\title{
Magnetic control of Leidenfrost drops
}

\author{
Keyvan Piroird, Christophe Clanet, and David Quéré* \\ PMMH, UMR 7636 du CNRS, ESPCI, 75005 Paris, France and LadHyX, UMR 7646 du CNRS, École Polytechnique, 91128 Palaiseau, France
}

(Received 1 March 2012; published 23 May 2012)

\begin{abstract}
We show how a magnetic field can influence the motion of a paramagnetic drop made of liquid oxygen in a Leidenfrost state on solids at room temperature. It is demonstrated that the trajectory can be modified in both direction and velocity and that the results can be interpreted in terms of classical mechanics as long as the drop does not get too close to the magnet. We study the deviation and report that it can easily overcome $180^{\circ}$ and even diverge under certain conditions, leading to situations where a drop gets captured. In the vicinity of the magnet, another type of trapping is observed, due to the deformation of the drop in this region, which leads to a strong energy dissipation. Conversely, drops can be accelerated by moving magnets (slingshot effect).
\end{abstract}

DOI: 10.1103/PhysRevE.85.056311

PACS number(s): 47.65.Cb, 47.55.D-, 75.50.Mm

A drop placed on a very hot solid levitates on its own vapor (Leidenfrost effect) [1-3]. Since there is no contact between the liquid and its substrate, friction is dramatically reduced: several meters are needed to observe the deceleration of millimetric Leidenfrost drops thrown on a horizontal surface at a few tens of $\mathrm{cm} / \mathrm{s}$ [4]. This extreme mobility makes levitating drops difficult to manipulate and to immobilize. Recent works have shown that it is possible to control the direction and velocity of Leidenfrost drops with asymmetric textured surfaces (ratchets) [5-8]. More generally, drop manipulation using external fields, such as mechanical vibrations [9], temperature [10], and electric [11,12] or magnetic fields, [13-15] has been the subject of numerous studies, but none of them involve Leidenfrost drops to our knowledge.

We propose to use magnetic fields to control the high mobility of Leidenfrost drops. For that purpose, we work with liquid oxygen, which is known to be paramagnetic [16]. Oxygen boils at $-183^{\circ} \mathrm{C}$ (at atmospheric pressure) and therefore undergoes the Leidenfrost effect on a substrate at room temperature, so we do not have to heat the substrate. We can imagine the same experiment with other magnetic fluids (such as ferrofluids or paramagnetic salt solutions) provided the substrate temperature is high compared to the boiling point of the liquid, and that the magnetic properties are not affected by heat, or by using a mechanism other than the Leidenfrost effect to reduce friction (with superhydrophobic surfaces, for example). In this article, we study how the motion of oxygen drops is influenced by the presence of a magnet. As we shall see, trajectories can be modified in direction and velocity, inducing situations where the drop can be captured by the magnet.

Liquid oxygen is obtained by distillation of air using liquid nitrogen, which boils at $-196^{\circ} \mathrm{C}$. A copper sheet of millimetric thickness is folded and welded to form a cone of about $10 \mathrm{~cm}$ in height and width. It is then filled with liquid nitrogen: the cone temperature reaches $-196^{\circ} \mathrm{C}$, that is to say, $13^{\circ} \mathrm{C}$ below the boiling point of oxygen present in the air, which therefore liquefies on the external surface of the cone. A film of liquid oxygen drains along this surface and drips at the tip, where it is recovered and directly used.

*Corresponding author: david.quere@espci.fr
To quantify how trajectories of liquid oxygen are modified by a magnetic field, the following experiment is made: an oxygen drop of typical radius $R=1 \mathrm{~mm}$ is thrown tangentially at a velocity $V$ on a horizontal glass plate of $10 \mathrm{~cm}$ of width and length, and $2 \mathrm{~mm}$ thick, below which we place a cylindrical neodymium magnet (grey circle in Fig. 1), $1 \mathrm{~cm}$ in diameter and height. The magnetic field is on the order of $0.5 \mathrm{~T}$ at the magnet and decreases on a distance comparable to its size. The drop is initially placed in an inclined gutter leading to the glass plate, which allows us, by changing the inclination, to vary $V$ between 20 and $60 \mathrm{~cm} / \mathrm{s}$. This velocity does not decrease by more than $10 \%$ when the drop crosses the glass plate.

With a magnet, as seen in the top views of Fig. 1, the trajectory of oxygen drops is deflected by an angle $\alpha$ relative to the incoming direction $\left[\alpha=30^{\circ}\right.$ in Fig. 1(a)]. The overall aspect of the trajectory evokes a hyperbola, as observed in central force systems. Trajectories can become more complex than conic curves: the deflection $\alpha$ is higher than $180^{\circ}$ in Fig. 1(b) and it exceeds $360^{\circ}$ in Figs. 1(c), and 1(d): drops can make more than a complete revolution around the magnet before escaping from its influence [Fig. 1(c)], showing that the liquid may be redirected in all possible directions. Similar trajectories are observed in basketball or golf, when a ball interacts with a hole $[17,18]$, which is due to the shape of the potential energy, as shown later. In addition, some drops are not able to escape and get trapped in an orbiting motion [Fig. 1(d)]. See the Supplementary Material [19] for illustrations of a variety of observed phenomena.

We measured the deflection $\alpha$ and the distance $r_{p}$ to the pericenter of the trajectory (where the radial velocity is equal to zero), as a function of $b$ for two fixed velocities: $V=22 \pm$ $2 \mathrm{~cm} / \mathrm{s}$ and $V=40 \pm 2 \mathrm{~cm} / \mathrm{s}$. As seen in Fig. 2(a), $\alpha$ is equal to zero for $b \rightarrow \infty$ and $b=0$, where a straight trajectory is indeed expected, and it has a maximum between these two limits. This maximum is high and sharp for $V=22 \mathrm{~cm} / \mathrm{s}$ : trajectories around $b=10 \mathrm{~mm}$ are highly deflected $(\alpha>$ $180^{\circ}$ ). At larger $V$, the maximum is smaller and smoother, suggesting that deflection results from a competition between kinetic and magnetic energies. In the same vein, Fig. 2(b) shows that $r_{p}$ approaches $b$ for $V=40 \mathrm{~cm} / \mathrm{s}$ whereas it can be twice smaller than $b$ for $V=22 \mathrm{~cm} / \mathrm{s}$ and $b=10 \mathrm{~mm}$, corresponding to situations where the deflection $\alpha$ is large. 


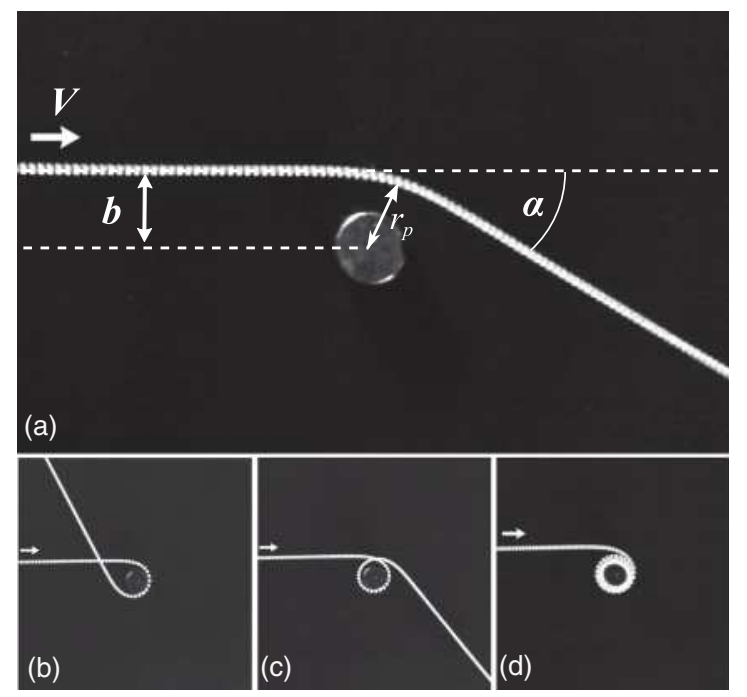

FIG. 1. Top views of oxygen drop trajectories on a horizontal glass plate below which a magnet (grey circle) of radius $5 \mathrm{~mm}$ is placed. (a) $V=25 \mathrm{~cm} / \mathrm{s}$ and $b=11 \mathrm{~mm}$. The trajectory bends by an angle $\alpha=30^{\circ}$. (b) $V=25 \mathrm{~cm} / \mathrm{s}$ and $b=9 \mathrm{~mm}$, we get a deflection $\alpha=245^{\circ}$. (c) $V=24 \mathrm{~cm} / \mathrm{s}$ and $b=9.5 \mathrm{~mm}, \alpha=410^{\circ}$ : the drop makes more than a full revolution around the magnet. (d) $V=$ $22 \mathrm{~cm} / \mathrm{s}$ and $b=10 \mathrm{~mm}$, the drop is trapped in an orbiting motion around the magnet.

The magnet exerts an attractive force on liquid oxygen, which derives from the magnetic energy per unit volume:

$$
E_{\text {mag }}=-\frac{\chi}{2 \mu_{o}} B^{2},
$$

where $\chi$ is the magnetic susceptibility of liquid oxygen $(\chi=$ 0.0035 at $\left.-183^{\circ} \mathrm{C}\right), \mu_{o}$ the magnetic permeability in vacuum $\left(4 \pi \times 10^{-7} \mathrm{H} / \mathrm{m}\right)$ and $B$ the magnetic field. Measuring $B$ in the horizontal plane with a teslameter gives the value of $E_{\text {mag }}$ as a function of the radial coordinate $r$ (inset of Fig. 3). Right above the magnet, the magnetic energy is ten times higher than gravity $\left(\rho g R \sim 10 \mathrm{~J} / \mathrm{m}^{3}\right.$ and $\left.\left|E_{\mathrm{mag}}\right| \sim 100 \mathrm{~J} / \mathrm{m}^{3}\right)$. Far from the magnet, $B$ is classically found to vary as $1 / r^{3}$, so that $\left|E_{\text {mag }}\right|$ falls off as $1 / r^{6}$. The whole magnetic potential can

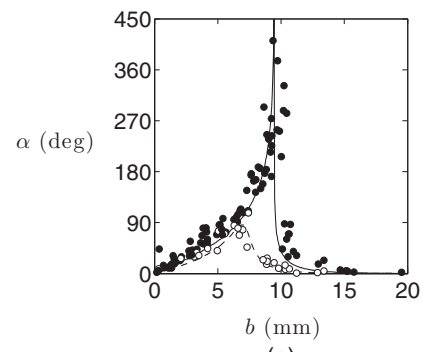

(a)

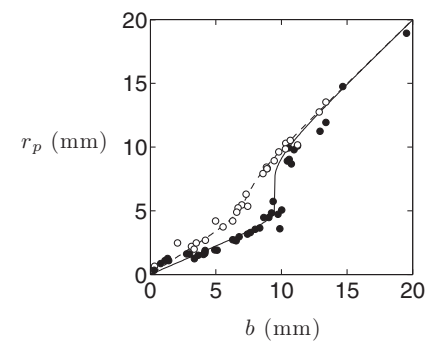

(b)
FIG. 2. (a) Angle of deflection $\alpha$ as a function of $b$ for two given velocities: $V=22 \pm 2 \mathrm{~cm} / \mathrm{s}(\bullet)$ and $V=40 \pm 2 \mathrm{~cm} / \mathrm{s}$ (०). Solid and dashed lines are calculations of $\alpha(b)$ with Eq. (4). (b) Distance $r_{p}$ between the magnet and the pericenter of the trajectory, as a function of $b$, for the same sets of data. The lines represent the model (see text) for $V=22 \mathrm{~cm} / \mathrm{s}$ (solid line) and $V=40 \mathrm{~cm} / \mathrm{s}$ (dashed line).

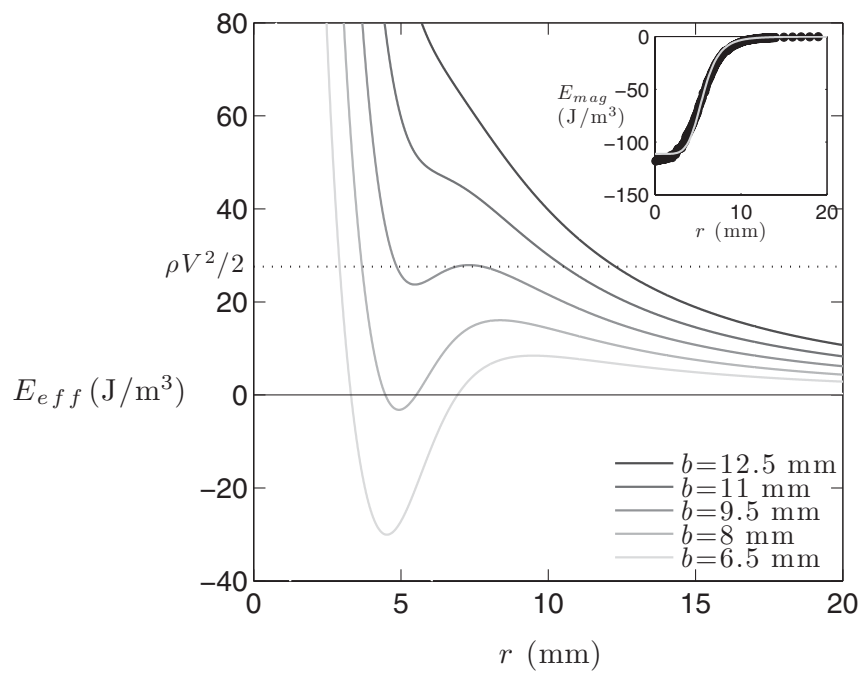

FIG. 3. Effective magnetic energy $E_{\text {eff }}$ per unit volume [defined after Eq. (3)] as a function of the drop-magnet distance $r$, for $V=$ $22 \mathrm{~cm} / \mathrm{s}$ and different impact parameters $b$. The dotted line represents the initial kinetic energy of the drop. The inset shows the measured magnetic energy around the magnet and its fit (grey line) by Eq. (2) used to calculate $E_{\text {eff }}(r)$.

eventually be written:

$$
E_{\mathrm{mag}}=-\frac{E_{o}}{q+\left(r / r_{o}\right)^{6}},
$$

with $E_{o}=9.41 \mathrm{~J} / \mathrm{m}^{3}, q=0.08$ and $r_{o}=8.5 \mathrm{~mm}$ (grey line in the inset of Fig. 3).

Knowing the magnetic energy at each point, we can calculate the trajectory of a drop and compare it to our measurements. We use polar coordinates $(r, \theta)$ to locate the drop relatively to the magnet center. We consider the drop as a point of density $\rho$, moving in a magnetic potential $E_{\mathrm{mag}}(r)$. Friction being extremely weak, we assume conservation of energy along the trajectory. Since the system is invariant by rotation, we can also write the conservation of angular momentum $\left(r^{2} \dot{\theta}=b V\right)$. Hence the equation of motion:

$$
\frac{\rho \dot{r}^{2}}{2}+E_{\text {eff }}(r)=\frac{\rho V^{2}}{2}
$$

where $E_{\text {eff }}(r)=\rho b^{2} V^{2} / 2 r^{2}+E_{\mathrm{mag}}(r)$ is the effective potential represented in Fig. 3 for a fixed velocity and different values of $b$. When $b$ is large, the magnetic energy is negligible and $E_{\text {eff }}$ monotonically decreases as $1 / r^{2}$. For small values of $b$, the presence of a magnetic term induces a local minimum and maximum in $E_{\text {eff }}(r)$. However, $E_{\mathrm{mag}}$ is always negligible compared to the orthoradial kinetic energy when $r \rightarrow 0$ and $r \rightarrow \infty$. Besides, since $E_{\mathrm{mag}}$ varies on a typical length-scale $\left|E_{\mathrm{mag}}(r) / E_{\mathrm{mag}}^{\prime}(r)\right| \geqslant 1.5 \mathrm{~mm}$ larger than the drop radius, the drop is assimilated to a point mass.

A first result obtained from $E_{\text {eff }}(r)$ is the position $r_{p}$ of the pericenter of the trajectory, given in Fig. 3 by the intersection of $E_{\text {eff }}(r)$ with the dotted line representing the total energy of the system $\rho V^{2} / 2$. If there are several intersections, the pericenter is the farthest from the magnet. When the magnetic energy is negligible, the trajectory is straight $\left(r_{p}=b\right)$. In the opposite limit, the drop is attracted by the magnet $\left(r_{p}<b\right)$. 
The calculation of $r_{p}(b)$ is in good agreement with the measurements made for each experiment, as seen in Fig. 2(b).

The deflection $\alpha$ can also be calculated by integrating $\dot{\theta}$ along the trajectory:

$$
\alpha=2 b \int_{r_{p}}^{+\infty} \frac{\mathrm{d} r}{r^{2} \sqrt{1-\frac{2 E_{\mathrm{eff}}(r)}{\rho V^{2}}}}-\pi .
$$

This equation is integrated numerically and the results, reported in Fig. 2(a), are in quantitative agreement with experiments without any adjustable parameter.

A singular behavior appears for $V \approx 22 \mathrm{~cm} / \mathrm{s}$ and $b \approx$ $10 \mathrm{~mm}$, where $r_{p}$ is discontinuous and deflection seems to diverge. A close look at the shape of the effective energy $E_{\text {eff }}(r)$ for these values of $V$ and $b$ (curve $b=9.5 \mathrm{~mm}$ in Fig. 3) reveals that the pericenter is located on top of the local maximum of $E_{\text {eff }}(r)$. This is an unstable equilibrium: radial velocity is equal to zero, as is radial acceleration since $E_{\mathrm{eff}}(r)$ is flat at this point. Such drops will "orbit" around the magnet, keeping a fixed radial distance and a constant angular velocity. This capture situation was indeed observed for those values of $V$ and $b$ [Fig. 1(d)]. This surprising trajectory is directly related to the presence of a local maximum in $E_{\text {eff }}(r)$, which can only exist if the potential decays faster than $1 / r^{2}$ as $r \rightarrow \infty$ (which is the case for a golf hole but not for a gravitational potential). For each value of $V$ lower than roughly $25 \mathrm{~cm} / \mathrm{s}$, there is an impact parameter $b$ for which the energy at the local maximum of $E_{\text {eff }}$ is equal to the initial energy, leading to a capture. For $V>25 \mathrm{~cm} / \mathrm{s}$, the kinetic energy is always higher than the maximum of $E_{\text {eff }}$ : capture becomes impossible. We thus obtain an ensemble of points $V(b)$ (dashed line in Fig. 4) for which orbital capture is predicted.

Figure 4 is a phase diagram reporting all observed capture situations ( $\boldsymbol{\nabla})$. For $r_{p}$ larger than the magnet size $R_{\text {mag }}$, capture

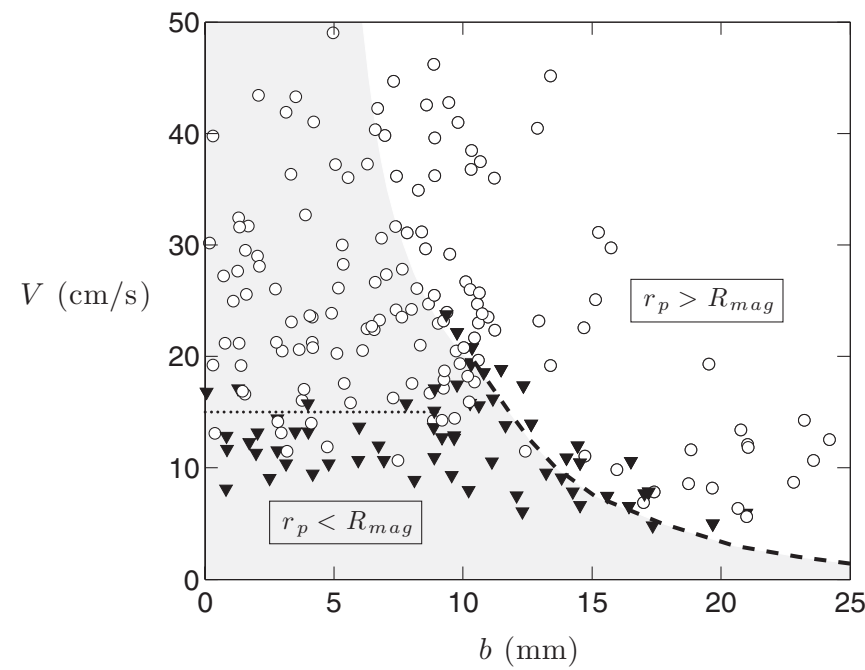

FIG. 4. Capture diagram. Each point represents an experiment: triangles $(\boldsymbol{\nabla})$ indicate drops that are captured above the magnet and circles (o) drops that escaped from its influence. The dashed line represents the values of $b$ and $V$ for which a capture is expected (see text). The dotted line represents critical velocity $V^{*}$ below which a drop passing above the magnet is trapped. The grey area indicates trajectories that pass above the magnet $\left(r_{p}<R_{\mathrm{mag}}\right)$. indeed occurs close to the dashed line corresponding to our model. At smaller distances $\left(r_{p}<R_{\text {mag }}\right)$, drops pass above the magnet and get captured below a critical velocity $V^{*} \approx$ $15 \mathrm{~cm} / \mathrm{s}$ (dotted line in Fig. 4), without orbiting motion. In these situations, drops are deformed by the magnet that acts as an enhanced gravity field, and energy conservation becomes questionable, as it is in impact phenomena where similar largescale deformations occur. Indeed, the energy needed to deform a drop of radius $R$ by an amount $\delta R \ll R$ is on the order of $8 \pi \gamma R \delta R$. As the drop moves away from the magnet, the deformation relaxes into vibrations that are eventually damped, resulting in an energy dissipation. This gives a critical velocity for which the drop loses all its kinetic energy:

$$
V^{*} \sim \sqrt{\frac{6 \gamma \delta R}{\rho R^{2}}} .
$$

In our experiments, we measure a typical deformation $\delta R \approx$ $0.3 \mathrm{~mm}$, which gives $V^{*} \sim 15 \mathrm{~cm} / \mathrm{s}$, consistent with our observations.

Magnets can finally be used to accelerate oxygen drops. The mechanism is inspired by the so-called gravity assist technique (or gravitational slingshot), exploiting the movement of planets to modify the speed of spacecrafts [20]. In the reference frame of the planet, a spacecraft coming at a velocity $V_{P}$ will leave at the same speed, but this is not true in another frame of reference, as shown in Figs. 5(a) and 5(b) by a simple composition of velocities.

To achieve a similar experiment, a thread is attached to the magnet and drawn at constant speed with a motor. Figures 5(c) and 5(d) show drops approaching a moving magnet, interacting with it and leaving in a direction influenced by that of the magnet. In both cases, the drop is accelerated. In the case of a

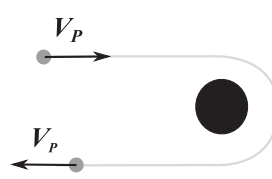

(a)

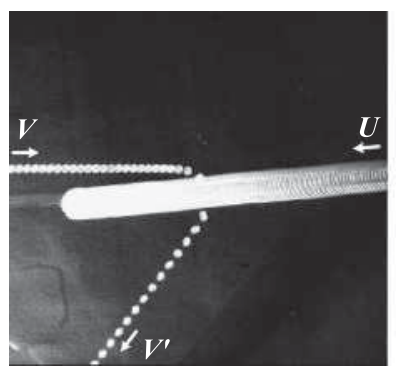

(c)

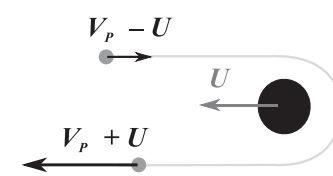

(b)

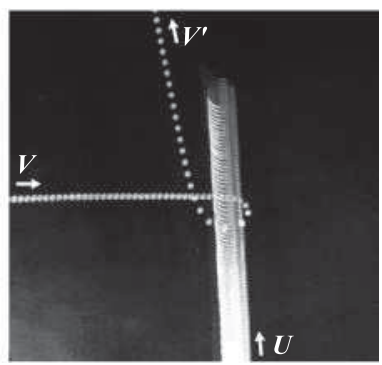

(d)
FIG. 5. (a) Elastic interaction between a planet and a light object in the reference frame of the planet. (b) Same collision in a reference frame where the planet moves at a velocity $U$. Here the light object leaves faster than it came. (c) Chronophotographs of drops moving at $V=12 \mathrm{~cm} / \mathrm{s}$ and passing above to a magnet traveling at a velocity $U=7 \mathrm{~cm} / \mathrm{s}$. Frontal approach: the drop is deflected by $125^{\circ}$ and leaves with a velocity $V^{\prime}=22 \mathrm{~cm} / \mathrm{s}$. (d) Lateral approach: the drop passes in front of the magnet, turns around it by $260^{\circ}$ and leaves at $V^{\prime}=18 \mathrm{~cm} / \mathrm{s}$. Time interval between photos: $20 \mathrm{~ms}$ in both figures. 
frontal approach [Fig. 5(c)], the final velocity $V^{\prime}$ of the drop is deduced by composition of velocities and conservation of energy during the interaction:

$$
V^{\prime}=-U \cos \alpha+\sqrt{(U+V)^{2}+U^{2}\left(\cos ^{2} \alpha-1\right)},
$$

where $U$ is the velocity of the magnet. $V^{\prime}$ is always greater than $V$ and is maximal for $\alpha=180^{\circ}$. For the experiment in Fig. 5(c) $\left(U=7 \mathrm{~cm} / \mathrm{s}, V=12 \mathrm{~cm} / \mathrm{s}\right.$, and $\left.\alpha=125^{\circ}\right)$, Eq. (6) predicts $V^{\prime}=22 \mathrm{~cm} / \mathrm{s}$, in agreement with the velocity of $21 \mathrm{~cm} / \mathrm{s}$ measured in this experiment. The same thing can be done for the lateral approach, for which we obtain

$$
V^{\prime}=-U \sin \alpha+\sqrt{V^{2}+U^{2} \sin ^{2} \alpha} .
$$

Here, $V^{\prime}=V$ if $\alpha=180^{\circ}$ since $V^{\prime}$ and $V$ are both orthogonal to $U$ in that case. If $\alpha>180^{\circ}$, the drop leaves in a direction similar to that of the magnet and it is accelerated $\left(V^{\prime}>V\right)$. For the situation of Fig. $5(\mathrm{~d})(U=7 \mathrm{~cm} / \mathrm{s}, V=12 \mathrm{~cm} / \mathrm{s}$, and $\alpha=260^{\circ}$ ), Eq. (7) predicts $V^{\prime}=20 \mathrm{~cm} / \mathrm{s}$, in agreement with the measurement of $18 \mathrm{~cm} / \mathrm{s}$. Finally, Eq. (7) predicts that $V^{\prime}$ should be less than $V$ for $\alpha<180^{\circ}$, giving another mechanism to slow down drops, and showing once again the versatility of this tool to manipulate, capture, slow down, or accelerate frictionless drops. In the same vein, it might be interesting to look at the effect of rotation, which can deform and even split a drop, and for which our system provides new possibilities for controlled experiments either by making a liquid orbit or by revolving the underlying magnet.
[1] J. G. Leidenfrost, De Aquae Communis Nonnullis Qualitatibus Tractatus (Duisburg, Germany, 1756).

[2] B. S. Gottfried, C. J. Lee, and K. J. Bell, Int. J. Heat Mass Transfer. 9, 1167 (1966).

[3] T. Tran, H. J. J. Staat, A. Prosperetti, C. Sun, and D. Lohse, Phys. Rev. Lett. 108, 036101 (2012).

[4] G. Dupeux, M. Le Merrer, C. Clanet, and D. Quéré, Phys. Rev. Lett. 107, 114503 (2011).

[5] H. Linke, B. J. Alemán, L. D. Melling, M. J. Taormina, M. J. Francis, C. C. Dow-Hygelund, V. Narayanan, R. P. Taylor, and A. Stout, Phys. Rev. Lett. 96, 154502 (2006).

[6] G. Lagubeau, M. Le Merrer, C. Clanet, and D. Quéré, Nat. Phys. 7, 395 (2011).

[7] J. T. Ok, E. Lopez-Oña, D. E. Nikitopoulos, H. Wong, and S. Park, Microfluid. Nanofluid. 10, 1045 (2011).

[8] T. R. Cousins, R. E. Goldstein, J. W. Jaworski, and A. I. Pesci, J. Fluid Mech. 696, 215 (2012).

[9] Y. Couder, S. Protière, E. Fort, and A. Boudaoud, Nature 437, 208 (2005).
[10] P. T. Nagy and G. P. Neitzel, Phys. Fluids 20, 101703 (2008).

[11] F. Mugele, Soft Matter 5, 3377 (2009).

[12] F. Mugele, M. Duits, and D. van den Ende, Adv. Colloid Interface Sci. 161, 115 (2009).

[13] J. R. Dorvee, A. M. Derfus, S. N. Bhatia, and M. J. Sailor, Nat. Mater. 3, 896 (2004).

[14] N.-T. Nguyen, K. M. Ng, and X. Huang, Appl. Phys. Lett. 89, 052509 (2006).

[15] Y. Zhao, J. Fang, H. Wang, X. Wang, and T. Lin, Adv. Mater. 22, 707 (2010).

[16] J. Dewar, Collected papers of Sir James Dewar (Cambridge University, Cambridge, England, 1927).

[17] H. Okubo and M. Hubbard, Sports Eng. 7, 15 (2004).

[18] B. W. Holmes, Am. J. Phys. 59, 129 (1991).

[19] See Supplemental Material at http://link.aps.org/supplemental/ 10.1103/PhysRevE.85.056311 for seven short movies illustrating the variety of observed phenomena.

[20] A. A. Bartlett and C. W. Hord, Phys. Teach. 23, 466 (1985). 